\title{
Antimicrobial Resistant Streptococcus pneumoniae
}

\author{
Khanal B, ${ }^{1}$ Acharya A, ${ }^{1}$ Amatya R, ${ }^{1}$ Gurung R, ${ }^{1}$ Poudyal N, ${ }^{1}$ Shrestha S, ${ }^{1}$ Bhattacharya SK ${ }^{1}$ \\ 'Department of Microbiology, BP Koirala Institute of Health Sciences, Dharan, Nepal.
}

\begin{abstract}
Introduction: Pneumococcal infections are important cause of morbidity and mortality. Knowledge of antimicrobial susceptibility patterns plays important role in the selection of appropriate therapy. Present study was undertaken to analyze the susceptibility patterns of pneumococcal isolates against commonly used antimicrobials with special reference to determination of minimum inhibitory concentration (MIC) of penicillin in a tertiary care hospital in eastern Nepal.
\end{abstract}

Methods: Twenty-six strains of S. pneumoniae isolated from various clinical specimens submitted to microbiology laboratory were evaluated. All isolates were tested for antimicrobial susceptibility by disk diffusion method. MIC of penicillin was tested by broth dilution method.

Results: Of the total isolates 19 (73\%) were from invasive infections. Seven isolates were resistant to cotrimoxazole. No resistance to penicillin was seen in disk diffusion testing. Less susceptibility to penicillin (MIC 0.1-1.0 mg/L) was observed in five (17\%) isolates. High level resistance to penicillin was not detected. One isolate was multidrug resistant.

Conclusions: S. pneumoniae isolates with intermediate resistance to penicillin prevail in Tertiary Care Hospital in eastern Nepal, causing invasive and noninvasive infections. As intermediate resistance is not detected in routine susceptibility testing, determination of MIC is important. It helps not only in the effective management of life threatening infections but is also essential in continuous monitoring and early detection of resistance. In addition, further study on pneumococcal infections, its antimicrobial resistance profile and correlation with clinical and epidemiological features including serotypes and group prevalence is recommended in future.

Keywords: antimicrobial susceptibility pattern, penicillin, Streptococcus pneumoniae

\section{INTRODUCTION}

Streptococcus pneumoniae, is associated most frequently with pneumonia, meningitis, bacteremia and many other infections, and continues to be an important cause of morbidity and mortality in developing countries. ${ }^{1-3}$ In our part of the world, empirical treatment still depends upon penicillin and other beta lactam antibiotics. However, with the rapid emergence of resistance, this is no longer a safe assumption. ${ }^{4}$ Trends indicate that pneumococci resistant to antibiotics are increasing worldwide. Therefore it may be expected that penicillin resistance may emerge sooner or later.

To determine the exact concentration of antibiotics required to inhibit the organism, determination of susceptibility by minimum inhibitory concentration (MIC) is preferred. This will also help to confirm the

\footnotetext{
Correspondence:

Dr. Basudha Khanal

Department of Microbiology

BPKIHS, Dharan, Nepal.

Email: basudhak@gmail.com

Phone: +977-25-525335
} 
Khanal et al. Antimicrobial Resistant Streptococcus pneumoniae in a Tertiary Care Hospital in Eastern Nepal

intermediate/ relative resistant which may indicate the development of impending resistance among local isolates. Present study was undertaken to analyze the susceptibility pattern of pneumococcal isolates with special reference to determination of MIC of penicillin.

\section{METHODS}

Cross-sectional study conducted at Microbiology unit of BP Koirala Institute of Health Sciences, Dharan, during April 2005 to April 2006, after obtaining approval from the research committee. S. pneumoniae isolates obtained from different clinical specimens submitted for culture sensitivity were included, duplicate sample from same patient were excluded. Gram stain of all samples except blood was done and cultured onto blood agar, chocolate agar and MacConkey agar medium. Pneumococci were identified by standard methods based on colony morphology, susceptibility to optochin $(5 \mu \mathrm{g})$ and bile solubility.5 Antimicrobial susceptibility test (AST) was performed according to the Clinical and Laboratory Standards Institute (CLSI) recommendations.6 Oxacillin $(1 \mu \mathrm{g})$ disc was used to screen for penicillin resistance. Cefotaxime $(30 \mu \mathrm{g})$, ciprofloxacin $(5 \mu \mathrm{g})$, chloramphenicol $(30 \mu \mathrm{g})$, cotrimoxazole $(1.25 / 23.75 \mu \mathrm{g})$, erythromycin $(15 \mu \mathrm{g})$ and vancomycin $5 \mu \mathrm{g})$ discs were used to perform the AST. MIC was determined by broth dilution method for penicillin according to the CLSI recommendations. ${ }^{7}$ Data were recorded in Ms Office EXCEL and percentage was calculated.

\section{RESULTS}

A total of 30 isolates of $S$. pneumoniae were obtained from the clinical specimen of 26 patients. These were from the cases of pneumonia (8), meningitis (9), septicemia (2) and infective keratitis (7). In two patients with meningitis pneumococcus was isolated simultaneously from blood and CSF. In other two patients with pneumonia two separate blood culture specimens yielded pneumococci (Table 1).

It was observed that the type of infection varied in different age groups. $50 \%$ of the patients with septicemia and meningitis were below five years whereas keratitis was common among adults and older age groups.

The youngest patient affected from septicemia and meningitis were 10 days and 60 days respectively. Age of patients with pneumonia ranged from 20-61 years. Sputum culture of the pneumonia patients did not yield the causative agents. Out of eight patients with pneumonia, pneumococci were recovered from blood in five and pleural fluid three.

Disc diffusion susceptibility testing showed that the isolates from normally sterile body sites were susceptible to antimicrobials other than cotrimoxazole. A total of six $(23.1 \%)$ strains were resistant to cotrimoxazole which included two isolates each from CSF and blood and one each from pleural fluid and corneal scraping. One isolate from the pleural fluid was also resistant to ciprofloxacin. No resistance to penicillin was observed by disc diffusion testing. Similarly all the isolates were susceptible to chloramphenicol, cefotaxime, erythromycin and vancomycin (Table 2).

Results of MIC determination showed that overall five $(17 \%)$ of the isolates had relative resistance to penicillin (Table 3). Of the invasive infections three $(9.5 \%)$ had

Table 1. Pattern of Pneumococcal infections in different age groups in BPKIHS hospital

\begin{tabular}{|c|c|c|c|c|c|}
\hline Infection & Age $<2$ months & 2 months-5yrs & $6-15$ yrs & $>15$ years & Total \\
\hline Meningitis & 1 & 5 & 1 & 2 & 9 \\
\hline Pneumonia & - & - & - & 8 & 8 \\
\hline Sepsis & 2 & - & - & - & 2 \\
\hline Keratitis & - & - & - & 7 & 7 \\
\hline Total & 3 & 5 & 1 & 17 & 26 \\
\hline
\end{tabular}

Table 2. Antimicrobial susceptibility pattern of $S$. pneumoniae in BPKIHS hospital on the basis of disc susceptibility testing

\begin{tabular}{cccccc}
\hline Antimicrobials & $\begin{array}{c}\text { CSF } \\
(\mathbf{n}=\mathbf{6})\end{array}$ & $\begin{array}{c}\text { Blood } \\
(\mathbf{n}=\mathbf{1 0})\end{array}$ & $\begin{array}{c}\text { Pleural fluid } \\
(\mathbf{n}=\mathbf{3})\end{array}$ & $\begin{array}{c}\text { Corneal } \\
\text { scraping } \\
(\mathbf{n}=\mathbf{7})\end{array}$ & $\begin{array}{c}\text { Total susceptibility } \\
(\mathbf{n}=\mathbf{2 6})\end{array}$ \\
\hline Cefotaxime & 6 & 10 & 3 & 7 & $26(100 \%)$ \\
Chloramphenicol & 6 & 10 & 3 & 7 & $26(100 \%)$ \\
Ciprofloxacin & 6 & 10 & 2 & 7 & $25(96 \%)$ \\
Cotrimoxazole & 4 & 8 & 2 & 6 & $20(76.9 \%)$ \\
\hline Erythromycin & 6 & 10 & 3 & 7 & $26(100 \%)$ \\
\hline Penicillin & 6 & 10 & 3 & 7 & $26(100 \%)$ \\
\hline Vancomycin & 6 & 10 & 3 & 7 & $26(100 \%)$ \\
\hline
\end{tabular}


Khanal et al. Antimicrobial Resistant Streptococcus pneumoniae in a Tertiary Care Hospital in Eastern Nepal

Table 3. MIC of Penicillin in S. pneumoniae isolates

\begin{tabular}{lcccc}
\hline Site of isolation & Total no of isolates & Penicillin MIC(mg/L) \\
\hline & & $<0.10$ & $0.1-1.0$ & $>1.0$ \\
CSF & 6 & 6 & 0 & 0 \\
Blood & 10 & 9 & 1 & 0 \\
Pleural fluid & 3 & 2 & 1 & 0 \\
Corneal ulcers & 7 & 4 & 3 & 0 \\
Total & 26 & 21 & 5 & 0 \\
\hline
\end{tabular}

reduced susceptibility to penicillin. These isolates were also resistant to cotrimoxazole. One isolate from pleural fluid which was multidrug resistant (cotrimoxazole, ciprofloxacin) required MIC of $0.125 \mu \mathrm{g} / \mathrm{ml}$. Penicillin MIC required for all the relative resistant pneumococci was $0.125 \mu \mathrm{g} / \mathrm{ml}$.

\section{DISCUSSION}

S. pneumoniae infections continue to impose a significant burden on human globally. With the introduction of polysaccharide pneumococcal vaccine, infections associated with this organism in the west are on the decline. However developing countries continue to bear the brunt of pneumococcal infections. The problem is further confounded with the emergence of antibiotic resistant pneumococci worldwide..$^{1-3,10-2}$

Penicillin has been the standard drug to treat pneumococcal infections for more than half a century. However, following the first report of penicillin resistance in 1967, there has been steady increase of resistant pneumococci. Rate of resistance varies geographically and largely reflect differing patterns of antimicrobial use. Worldwide, rate of penicillin resistance among pneumococci has been changing. According to a study by Asian network on surveillance of resistant pathogen (ANSORP), the frequencies of nonsusceptible pneumococcal strains to penicillin was as high as $79.7 \%$ in Korea and as low as $3.8 \%$ in India. ${ }^{11}$ Cleveland clinic reported the steady development of resistance to penicillin among invasive isolates of pneumococci over a span of 25 years. ${ }^{12}$ Penicillin resistance was detected in $64 \%$ isolates obtained from pediatric age group in a study in China. ${ }^{13}$ In a small study conducted at our centre in $2001,4 \%$ of the total isolates were found to be penicillin resistant but all the invasive isolates were susceptible to penicillin.

On the basis of breakpoints determined by the Clinical Laboratory Standard Institute (CLSI) penicillin resistant pneumococcusisreported to beeitherintermediate/relative or high level resistant. ${ }^{7-9}$ Prevalence of pneumococcal strains with intermediate/relative resistance MIC 0.1$1.0 \mathrm{mg} / \mathrm{L}$ has increased at an alarmingly rate worldwide in the past decade. According to a recent surveillance data, the worldwide prevalence of $S$. pneumoniae with reduced susceptibility to penicillin is $18.2 \% .{ }^{14} \mathrm{~A}$ study from north India too revealed the presence of $18.3 \%$ of clinical isolates and $16 \%$ of nasopharyngeal isolates possessing reduced susceptibility to penicillin. ${ }^{15}$ In the present study, $17 \%$ of the total isolates had relative resistance to penicillin, which included two (7.6\%) strains from invasive infections. Our finding is similar to that of others though the number of isolates were less. ${ }^{14,15}$

High level penicillin resistant pneumococci with MIC $2 \mathrm{mg} / \mathrm{L}$ have also emerged and are becoming more common in certain geographical area. These strains are also observed to be multidrug resistant, thus posing potential threats in the treatment of pneumococcal diseases.9,14 Pneumococci with high level penicillin resistance were not encountered in the present study. However, one strain was multidrug resistant, exhibiting resistance to cotrimoxazole, ciprofloxacin, and relative resistance to penicillin. Its prevalence among the invasive isolates is similar to findings from other reports from Indian subcontinent. ${ }^{10,15,16}$ In the present study, prevalence of resistance to cotrimoxazole $(23.1 \%)$ was low as compared to prevalence reported in other studies including one conducted in Nepal. ${ }^{15,16}$

It could be due to infrequent use of cotrimoxazole in this part. High level resistance to penicillin was not observed in the present study. Relative resistance to penicillin was also not detected in the routinely performed disc susceptibility testing. It underscores the importance of determination of MIC. MIC testing is important not only for the effective management of this potentially life threatening infection but also for continuous monitoring and early detection of resistance. MIC determination technique performed in the present study is economical but cumbersome. Considering the serious nature of the infection and importance of prompt report, the methods for the rapid detection of MIC (eg. E-test) is desirable for the timely optimization of the therapy.

Since the first documentation of resistance strains of pneumococcus, there has been rapid increase and spread of resistance all over. Because pneumococci are part of the normal commensal flora they are constantly under pressure and are likely to acquire resistance to antibiotics given for various other infections. Considering the relative resistance among pneumococci in the present study, refraining from the use of antibiotics in 
Khanal et al. Antimicrobial Resistant Streptococcus pneumoniae in a Tertiary Care Hospital in Eastern Nepal

mild self limiting upper respiratory tract infections is important to limit the spread of resistance.

Invasive pneumococcal infections which are significant cause of morbidity and mortality are prevalent in our part of the world. In addition, increasing penicillin and multidrug resistance among pneumococci has important clinical impact. High dose penicillin $G$ and many other agents continue to be efficacious potentially for pneumonia and bacteremia. However, treatment options for meningitis and for certain pediatric infections which are treated with oral agents have been limited by resistance. ${ }^{17}$ Factors independent of antimicrobial susceptibility of infecting organisms are also thought to have significant impact on clinical outcome in severe pneumococcal diseases. Host factors such as extremes of age, underlying immune status or debilitation, comorbid conditions or microbial virulence factors such as capsular serotypes strongly influence the prognosis. ${ }^{18}$

Yield of pneumococci from the clinical specimens was not considered optimum in the present study as most of the patients visit this tertiary care hospital after receiving treatment in other centers, which in many situations would have inhibited the organism but not eradicated them leading to unresponsiveness to therapy and failure in isolation of organism in culture. Due to lack of facilities serotyping could not be attempted. Prevalence of serogroup /type in this area has not been studied so far. As the present study reveals the laboratory based results, further study on pneumococcal infections, its antimicrobial resistance profile and correlation with clinical and epidemiological features including serotype /serogroup prevalence is recommended in future.

\section{CONCLUSIONS}

Pneumococci, being a commensal flora are constantly under pressure and are likely to acquire resistance to antibiotics given for various other infections. Penicillin resistant pneumococci prevail in our setup though invasive infections are still caused by the susceptible kind. Considering the relative resistance among pneumococci as observed in the present study, judicious use of antibiotics in upper respiratory tract infections is important to limit the development and spread of high level resistance to penicillin among clinical isolates of pneumococci. Moreover, as routinely performed disc susceptibility testing was not able to detect the relative resistance to penicillin, our study underscores importance of determination of MIC in laboratory. As the present study reveals the laboratory based results, further study on pneumococcal infections, its antimicrobial resistance profile and correlation with clinical and epidemiological features including serotype/serogroup prevalence is recommended in future.

\section{ACKNOWLEDGEIMENTS}

The study was supported by the B.P. Koirala institute of Health Sciences, Dharan. The authors thank Mr. Ram Babu Yadav for his technical assistance.

\section{REFERENCES}

1. Klugman KP, Tuomanen E. Pneumococcal infections. In: Guerrant RL, Walker DH, Weller PF, eds. Tropical Infectious Diseases. Principles, pathogens and practice: Philadelphia; Churchill Livingstone: 1999. p. 401-7.

2. Austrian R. Streptococcus pneumoniae. In: Gorbach SL, Bartlett JG, Blacklow NR, eds. Infectious Diseases: Philadelphia; WB Saunders Company: 1999. p. 1719-23.

3. Musher DM. Streptococcus pneumoniae. In: Mandell GL, Bennett JE, Dolin R, eds. Principles and Practice of Infectious diseases: New York; Churchill Livingstone: 2000. p. 2128-47.

4. Appelbaum PC. Antimicrobial resistance in Streptococcus pneumoniae: an overview. Clin Infect Dis. 1992;15:77-83.

5. Ross PW. Streptococcus pneumoniae. In: Collee JG, Fraser AG, Marmion BP, Simmons A, eds. Mackie \& McCartney Practical Medical Microbiology. 14th ed. Churchill Livingstone: 1996. p. 275-81.

6. Clinical and laboratory Standards Institute. Performance standards for antimicrobial disc susceptibility tests. 9th ed. Approved Standard. Wayne, A: Clinical and Laboratory Standards Institute: 2006. (CLSI document no M2-A9)
7. Clinical and Laboratory Standards Institute. Methods for Dilution Antimicrobial Susceptibility Tests for Bacteria that grow aerobically. 7th ed. Approved standard. Wayne, PA: Clinical and Laboratory Standards Institute: 2006. (CLSI document no M7-A7)

8. Kanungo R, D'Lima D, Rajalakshmi B, Kumar A, Badrinath S. Emerging antibiotic resistant pneumococci in invasive infections in South India: need for monitoring. Ind J Pharmacol: 2002. 34:38-43.

9. Song JH, Lee NY, Ichiyama S, et al. Spread of drug resistant Streptococcus pneumoniae in Asian countries: Asian Network for Surveillance of Resistant Pathogens (ANSORP) study. Clin Infect Dis. 1999;28(6):1206-11.

10. Jacobs MR, Good CE, Beall B, Bajaksouzian S, Windau AR, Whitney CG. Changes in serotype and antimicrobial susceptibility of invasive Streptococcus pneumoniae strains in Cleveland: a quarter century of experience. J Clin Microbiol. 2008;46(3):982-90.

11. Liu Y, Wang H, Chen M, et al. Serotype distribution and antimicrobial resistance patterns of Streptococcus pneumoniae isolated from children in China younger than 5 years. Diagn Microbiol Infect Dis. 2008;61(3):256-63. 
Khanal et al. Antimicrobial Resistant Streptococcus pneumoniae in a Tertiary Care Hospital in Eastern Nepal

12. Jacobs MR. Worldwide trends in antimicrobial resistance among common respiratory tract pathogens in children. Pediatric Infect Dis J. 2003;22(8):109-19.

13. Goyal R, Singh NP, Kaur M, Talwar V. Antimicrobial resistance in invasive and colonizing Streptococcus pneumoniae in North India. Ind J Med Microbiol. 2007;25(3):256-9.

14. Shah AS, Knoll MD, Sharma PR, et al. Invasive pneumococcal disease in Kanti Children's Hospital, Nepal as observed by South Asian Pneumococcal Alliance Network. Clin Infect Dis. 2009;48:123-8.
15. Jacobs MR. Antimicrobial resistant Streptococcus pneumoniae: trends and management. Exper Rev Anti Infect Ther. 2008;6(5):619-35.

16. Lynch JP, Zhanel GG. Streptococcus pneumoniae: does antimicrobial resistance matter? Semin Respir Crit Care Med. 2009;30(2):210-38. 